\title{
STABILITY AND HOPF BIFURCATION ANALYSIS FOR A LOTKA-VOLTERRA PREDATOR-PREY MODEL WITH TWO DELAYS
}

\author{
Changuin XU* ${ }^{*}$, MaOXin LiAO ${ }^{*, * *}$, XiaOfei HE*,*** \\ * (i) School of Mathematics and Statistics \\ Guizhou College of Finance and Economics, Luchongguan Rd 269, Guiyang 550004, PR China \\ (ii) Faculty of Science \\ Hunan Institute of Engineering, Fuxing Rd 88, Xiangtan 411004, PR China \\ e-mail: $\mathrm{xcj} 403 \mathrm{a} 126 . \mathrm{com}$ \\ ${ }^{* *}$ School of Mathematics and Physics \\ Nanhua University, Changsheng Rd 26, Hengyang 421001, PR China \\ e-mail: maoxinliao@163.com \\ *** Department of Mathematics \\ Zhangjiajie College of Jishou University, Renming Rd 120, Zhangjiajie 427000, PR China \\ e-mail: hexiaofei525@163.com
}

\begin{abstract}
In this paper, a two-species Lotka-Volterra predator-prey model with two delays is considered. By analyzing the associated characteristic transcendental equation, the linear stability of the positive equilibrium is investigated and Hopf bifurcation is demonstrated. Some explicit formulae for determining the stability and direction of Hopf bifurcation periodic solutions bifurcating from Hopf bifurcations are obtained by using normal form theory and center manifold theory. Some numerical simulations for supporting the theoretical results are also included.
\end{abstract}

Keywords: predator-prey model, delay, stability, Hopf bifurcation.

\section{Introduction}

Various mathematical models have been established in the study of populations since Vito Volterra and James Lotka proposed seminal models of predator-prey models in the mid 1920s. Among these models, predator-prey models play an important role in population dynamics. Many theoreticians and experimentalists concentrated on the stability of predator-prey systems and, more specifically, they investigated the stability of such systems when time delays are incorporated into the models. Such delayed systems received great attention since time delay may have very complicated impact on the dynamical behavior of the system such as the periodic structure, bifurcation, etc. (Kuang and Takeuchi, 1994; Xu et al., 2004; Zhou et al., 2008; Teramoto et al., 1979; Bhattacharyya and Mukhopadhyay, 2006; Prajneshu Holgate, 1987; Gao et al., 2008; Xu and Ma, 2008; Kar and Pahari, 2007; Klamka, 1991).

May (1973) first proposed and briefly discussed the stability of the following delayed predator-prey system:

$$
\left\{\begin{array}{l}
\dot{x}(t)=x(t)\left[r_{1}-a_{11} x(t-\tau)-a_{12} y(t)\right], \\
\dot{y}(t)=y(t)\left[-r_{2}+a_{21} x(t)-a_{22} y(t)\right],
\end{array}\right.
$$

where $x(t)$ and $y(t)$ can be interpreted as the population densities of preys and predators at time $t$, respectively; $\tau \geq 0$ is the feedback time delay of the preys to the growth of the species itself; $r_{1}>0$ denotes an intrinsic growth rate of the preys and $r_{2}>0$ denotes the death rate of the predators; the parameters $a_{i j}(i, j=1,2)$ are all positive constants.

Song and Wei (2005) investigated further the dynamics of the system (1). Considering the delay $\tau$ as the bifurcation parameter, they obtained that, under certain conditions, the unique positive equilibrium of the system (1) is absolute stable while it is conditionally stable and there exist $k$ switches from stability to instability to stability under other conditions. Further, by using normal form theory and the center manifold theorem, they obtained the formu- 
lae for determining the direction of Hopf bifurcations and the stability of bifurcating periodic solutions.

Yan and Li (2006) incorporated the same delay $\tau$ into the population density of the predator in the second equation of the system (1) and obtained the following:

$$
\left\{\begin{array}{l}
\dot{x}(t)=x(t)\left[r_{1}-a_{11} x(t-\tau)-a_{12} y(t)\right], \\
\dot{y}(t)=y(t)\left[-r_{2}+a_{21} x(t)-a_{22} y(t-\tau)\right],
\end{array}\right.
$$

Regarding the delay $\tau$ as the bifurcation parameter, they investigated the stability of the system (2) and studied the properties of Hopf bifurcation for the system (2) by using normal form theory and the center manifold theorem, which is different from that used by Song and Wei (2005).

Faria (2001) investigated the stability and Hopf bifurcation of the following system with two different delays:

$$
\left\{\begin{array}{l}
\dot{x}(t)=x(t)\left[r_{1}-a_{11} x(t)-a_{12} y\left(t-\tau_{2}\right)\right], \\
\dot{y}(t)=y(t)\left[-r_{2}+a_{21} x\left(t-\tau_{1}\right)-a_{22} y(t)\right] .
\end{array}\right.
$$

According to the view point of Kuang (1993), Yan and Zhang (2008) considered the stability and Hopf bifurcation of the following delayed system:

$$
\left\{\begin{array}{l}
\dot{x}(t)=x(t)\left[r_{1}-a_{11} x(t-\tau)-a_{12} y(t-\tau)\right], \\
\dot{y}(t)=y(t)\left[-r_{2}+a_{21} x(t-\tau)-a_{22} y(t-\tau)\right] .
\end{array}\right.
$$

Based on (1)-(4), we consider the following system:

$$
\left\{\begin{array}{l}
\dot{x}(t)=x(t)\left[r_{1}-a_{11} x\left(t-\tau_{1}\right)-a_{12} y\left(t-\tau_{2}\right)\right], \\
\dot{y}(t)=y(t)\left[-r_{2}+a_{21} x\left(t-\tau_{2}\right)-a_{22} y\left(t-\tau_{1}\right)\right],
\end{array}\right.
$$

where $x(t)$ and $y(t)$ denote the population densities of preys and predators at time $t$, respectively; $r_{1}>0$ denotes the intrinsic growth rate of preys and $r_{2}>0$ denotes the death rate of predators; the parameters $a_{i j}(i, j=1,2)$ are all positive constants; $\tau_{1}$ is the gestation periodic of preys and predators; $\tau_{2}$ in the first equation of the system (5) denotes the hunting delay of predator to prey and $\tau_{2}$ in the second equation of the system (5) is the delay in predator maturation.

The biological meaning of the system (5) is as follows. In the absence of predators, the prey species follows the logistic equation

$$
\dot{x}(t)=x(t)\left[r_{1}-a_{11} x\left(t-\tau_{1}\right)\right] .
$$

In the presence of predators, there is a hunting term, $a_{12} y\left(t-\tau_{2}\right)$, with a certain delay $\tau_{2}$ called the hunting delay. In the absence of prey species, the predator species follows the equation

$$
\dot{y}(t)=x(t)\left[-r_{2}-a_{22} y\left(t-\tau_{1}\right)\right],
$$

i.e., the number of predators decreases. The positive feedback $a_{21} x\left(t-\tau_{2}\right)$ has a positive delay $\tau_{2}$ which is the delay in predator maturation.
We would like to point out that the systems (1)-(4) are all a special case of the system (5). In this paper, we will study the stability, and local Hopf bifurcation for the system (5). To the best of our knowledge, it is the first time the research of Hopf bifurcation for the model (5) is undertaken.

The remainder of the paper is organized as follows. In Section 2, we investigate the stability of the positive equilibrium and the occurrence of local Hopf bifurcations. In Section 3, the direction and stability of local Hopf bifurcation are established. In Section 4, numerical simulations are carried out to illustrate the validity of the main results. Some main conclusions are drawn in Section 5.

\section{Stability of the positive equilibrium and local Hopf bifurcations}

In this section, we shall focus on analyzing the corresponding linearized system at the positive equilibrium of the system (5) and investigate the stability of this equilibrium point and the existence of local Hopf bifurcations occurring at the positive equilibrium.

Since any time delay does not change the equilibrium of the system and, according to Yan and Zhang (2008), we know that the delayed prey predator model (5) has unique positive equilibrium points $E_{0}\left(x^{*}, y^{*}\right)$, where

$$
x^{*}=\frac{r_{1} a_{22}+r_{2} a_{12}}{a_{11} a_{22}+a_{12} a_{21}}, \quad y^{*}=\frac{r_{1} a_{21}-r_{2} a_{11}}{a_{11} a_{22}+a_{12} a_{21}},
$$

if

$$
\text { (H1) } \quad r_{1} a_{21}-r_{2} a_{11}>0 .
$$

Let $\bar{x}(t)=x(t)-x^{*}, \bar{y}(t)=y(t)-y^{*}$ and still denote $\bar{x}(t), \bar{y}(t)$ by $x(t), y(t)$, respectively. Then (5) becomes

$$
\left\{\begin{aligned}
\dot{x}(t)= & m_{1} x(t)+m_{2} x\left(t-\tau_{1}\right)+m_{3} y\left(t-\tau_{2}\right) \\
& +m_{4} x(t) x\left(t-\tau_{1}\right)+m_{5} x(t) y\left(t-\tau_{2}\right), \\
\dot{y}(t)= & n_{1} y(t)+n_{2} x\left(t-\tau_{2}\right)+n_{3} y\left(t-\tau_{1}\right) \\
& +n_{4} x\left(t-\tau_{2}\right) y(t)+n_{5} y(t) y\left(t-\tau_{1}\right),
\end{aligned}\right.
$$

where

$$
\begin{aligned}
m_{1} & =r_{1}-a_{11} x^{*}-a_{12} y^{*}, & m_{2} & =-a_{11} x^{*}, \\
m_{3} & =-a_{12} x^{*}, m_{4}=-a_{12}, & m_{5} & =-a_{12}, \\
n_{1} & =-r_{2}+a_{21} x^{*}-a_{22} y^{*}, & n_{2} & =a_{21} y^{*}, \\
n_{3} & =-a_{22} y^{*}, n_{4}=a_{21}, & n_{5} & =-a_{22} .
\end{aligned}
$$

It is easy to check that $m_{1}=n_{1}=0$. The linearization of Eqn. $(6)$ at $(0,0)$ is

$$
\left\{\begin{array}{l}
\dot{x}(t)=m_{2} x\left(t-\tau_{1}\right)+m_{3} y\left(t-\tau_{2}\right), \\
\dot{y}(t)=n_{2} x\left(t-\tau_{2}\right)+n_{3} y\left(t-\tau_{1}\right),
\end{array}\right.
$$


whose characteristic equation is

$$
\begin{aligned}
\lambda^{2}-\left(m_{2}+\right. & \left.n_{3}\right) \lambda e^{-\lambda \tau_{1}} \\
& +m_{2} n_{3} e^{-2 \lambda \tau_{1}}-m_{3} n_{2} e^{-2 \lambda \tau_{2}}=0
\end{aligned}
$$

In order to investigate the distribution of roots of the transcendental equation (8), the following result is useful.

Lemma 1. (Ruan and Wei, 2003) For the transcendental equation

$$
\begin{aligned}
P(\lambda, & \left.e^{-\lambda \tau_{1}}, \ldots, e^{-\lambda \tau_{m}}\right) \\
= & \lambda^{n}+p_{1}^{(0)} \lambda^{n-1}+\cdots+p_{n-1}^{(0)} \lambda+p_{n}^{(0)} \\
& +\left[p_{1}^{(1)} \lambda^{n-1}+\cdots+p_{n-1}^{(1)} \lambda+p_{n}^{(1)}\right] e^{-\lambda \tau_{1}}+\cdots \\
& +\left[p_{1}^{(m)} \lambda^{n-1}+\cdots+p_{n-1}^{(m)} \lambda+p_{n}^{(m)}\right] e^{-\lambda \tau_{m}}=0,
\end{aligned}
$$

as $\left(\tau_{1}, \tau_{2}, \tau_{3}, \ldots, \tau_{m}\right)$ vary, the sum of orders of the zeros of $P\left(\lambda, e^{-\lambda \tau_{1}}, \ldots, e^{-\lambda \tau_{m}}\right)$ in the open right half plane can change, and only a zero appears on or crosses the imaginary axis.

In the sequel, we consider three cases.

Case A: $\tau_{1}=\tau_{2}=0$. Then (8) becomes

$$
\lambda^{2}-\left(m_{2}+n_{3}\right) \lambda+m_{2} n_{3}-m_{3} n_{2}=0 .
$$

A set of necessary and sufficient conditions that all roots of (9) have a negative real part is given in the following form:

$$
\text { (H2) } \quad m_{2}+n_{3}<0, \quad m_{2} n_{3}-m_{3} n_{2}>0 .
$$

Then the equilibrium point $E_{0}\left(x^{*}, y^{*}\right)$ is locally asymptotically stable when the condition $(H 1)$ holds.

Case B: $\tau_{1}=0, \tau_{2}>0$. Then (8) becomes

$$
\lambda^{2}+p \lambda+r+q e^{-2 \lambda \tau_{2}}=0,
$$

where

$$
p=-\left(m_{2}+n_{3}\right), \quad r=m_{2} n_{3}, \quad q=-m_{3} n_{2} .
$$

For $\omega>0, i \omega$ being a root of (10), it follows that

$$
\left\{\begin{array}{l}
q \cos 2 \omega \tau_{2}=\omega^{2}-r \\
q \sin 2 \omega \tau_{2}=p \omega
\end{array}\right.
$$

which leads to

$$
\omega^{4}+\left(p^{2}-2 r\right) \omega^{2}+r^{2}-q^{2}=0 .
$$

It is easy to see that if the condition

$$
\text { (H3) } \quad p^{2}-2 r>0, \quad r^{2}-q^{2}>0
$$

holds, then Eqn. (12) has no positive roots. Hence, all roots of (10) have negative real parts when $\tau_{2} \in[0,+\infty)$ under the conditions $(H 2)$ and $(H 3)$.

If $(H 2)$ and

$$
\text { (H4) } p^{2}-2 r>0, \quad r^{2}-q^{2}<0
$$

hold, then (12) has a unique positive root $\omega_{0}^{2}$. Substituting $\omega_{0}^{2}$ into (11), we obtain

$$
\tau_{2_{n}}=\frac{1}{2 \omega_{0}}\left\{\arccos \frac{q\left(\omega_{0}^{2}-r\right)}{q^{2}}+2 n \pi\right\},
$$

$n=0,1,2, \ldots$.

Let $\lambda\left(\tau_{2}\right)=\alpha\left(\tau_{2}\right)+i \omega\left(\tau_{2}\right)$ be a root of (10) near $\tau_{2}=\tau_{2_{n}}$ and $\alpha\left(\tau_{2_{n}}\right)=0, \omega\left(\tau_{2_{n}}\right)=\omega_{0}$. From functional differential equation theory, for every $\tau_{2_{n}}, n=$ $0,1,2, \ldots$, there exists $\varepsilon>0$ such that $\lambda\left(\tau_{2}\right)$ is continuously differentiable at $\tau_{2}$ for $\left|\tau_{2}-\tau_{2_{n}}\right|<\varepsilon$. Substituting $\lambda\left(\tau_{2}\right)$ into the left-hand side of (10) and differentiating with respect to $\tau_{2}$, we have

$$
\left(\frac{\mathrm{d} \lambda}{\mathrm{d} \tau_{2}}\right)^{-1}=\frac{(2 \lambda+p) e^{2 \lambda \tau_{2}}}{2 q \lambda}-\frac{\tau_{2}}{\lambda},
$$

which leads to

$$
\begin{aligned}
{\left[\frac{\mathrm{d}(\operatorname{Re} \lambda(\tau))}{\mathrm{d} \tau_{2}}\right]_{\tau_{2}=\tau_{2_{n}}}^{-1} } & =\left.\operatorname{Re}\left\{\frac{(2 \lambda+p) e^{2 \lambda \tau_{2}}}{2 q \lambda}\right\}\right|_{\tau_{2}=\tau_{2_{n}}} \\
& =\frac{p \sin 2 \omega_{0} \tau_{2_{n}}+2 \omega_{0} \cos 2 \omega_{0} \tau_{2_{n}}}{q \omega_{0}} \\
& =\frac{p^{2}-2 r+2 \omega_{0}^{2}}{2 q^{2}}>0 .
\end{aligned}
$$

Noting that

$$
\begin{aligned}
\left.\operatorname{sign}\left\{\frac{\mathrm{d}(\operatorname{Re} \lambda)}{\mathrm{d} \tau_{2}}\right\}\right|_{\tau_{2}=\tau_{2_{n}}} & \\
& =\left.\operatorname{sign}\left\{\operatorname{Re}\left(\frac{\mathrm{d} \lambda}{\mathrm{d} \tau_{2}}\right)\right\}\right|_{\tau_{2}=\tau_{2_{n}}}=1
\end{aligned}
$$

we have

$$
\left.\frac{\mathrm{d}(\operatorname{Re} \lambda)}{\mathrm{d} \tau_{2}}\right|_{\tau_{2}=\tau_{2_{n}}}>0 .
$$

According to the above analysis and Corollary 2.4 of Ruan and Wei (2003), we have the following results.

Lemma 2. For $\tau_{1}=0$, assume that $(H 1)$ and $(H 2)$ are satisfied. Then the following conclusions hold:

(i) If (H3) holds, then the positive equilibrium $E_{0}\left(x^{*}, y^{*}\right)$ of the system (5) is asymptotically stable for all $\tau_{2} \geq 0$.

(ii) If (H4) holds, then the positive equilibrium $E_{0}\left(x^{*}, y^{*}\right)$ of the system (5) is asymptotically stable for $\tau_{2}<\tau_{2_{0}}$ and unstable for $\tau_{2}>\tau_{2_{0}}$. Furthermore, the system (5) undergoes a Hopf bifurcation at the positive equilibrium $E_{0}\left(x^{*}, y^{*}\right)$ when $\tau_{2}=\tau_{2_{0}}$. 
Case $C: \tau_{1}>0, \tau_{2}>0$. We consider Eqn. (8) with $\tau_{2}$ in its stable interval. Regarding $\tau_{1}$ as a parameter, without loss of generality, we consider the system (5) under Assumptions $(H 2)$ and $(H 4)$. Let $i \omega(\omega>0)$ be a root of (8). Then we can obtain

$$
k_{1} \omega^{4}+k_{2} \omega^{3}+k_{3} \omega^{2}+k_{4}=0,
$$

where

$$
\begin{aligned}
k_{1}= & 2\left(m_{2} n_{3}+\cos 2 \omega \tau_{2}\right)^{2}+\sin ^{2} 2 \omega \tau_{2}, \\
k_{2}= & -2\left(m_{2}+n_{3}\right) \sin 2 \omega \tau_{2}\left[m_{3} n_{2}\left(m_{2} n_{3}+\cos 2 \omega \tau_{2}\right)\right. \\
& \left.+m_{2} n_{3}-m_{3} n_{2} \cos 2 \omega \tau_{2}\right], \\
k_{3}= & {\left[\left(m_{2}+n_{3}\right) m_{3} n_{2} \sin 2 \omega \tau_{2}\right]^{2}+\left[( m _ { 2 } + n _ { 3 } ) \left(m_{2} n_{3}\right.\right.} \\
& \left.\left.-m_{3} n_{2} \cos 2 \omega \tau_{2}\right)\right]^{2}, \\
k_{4}= & -\left[\left(m_{2} n_{3}-m_{3} n_{2} \cos 2 \omega \tau_{2}\right)\left(m_{2} n_{3}+\cos 2 \omega \tau_{2}\right)\right. \\
& \left.+m_{3} n_{2} \sin ^{2} 2 \omega \tau_{2}\right]^{2} .
\end{aligned}
$$

Write

$$
H(\omega)=k_{1} \omega^{4}+k_{2} \omega^{3}+k_{3} \omega^{2}+k_{4} .
$$

It is easy to check that $H(0)<0$ and $\lim _{\omega \rightarrow+\infty} H(\omega)=$ $+\infty$. We can obtain that (15) has finite positive roots $\omega_{1}, \omega_{2}, \ldots, \omega_{n}$. For every fixed $\omega_{i}, i=1,2,3, \ldots, k$, there exists a sequence $\left\{\tau_{1_{i}}^{j} \mid j=1,2,3, \ldots\right\}$, such that (15) holds. Let

$$
\tau_{1_{0}}=\min \left\{\tau_{1_{i}}^{j} \mid i=1,2, \ldots, k ; \quad j=1,2, \ldots\right\} .
$$

When $\tau_{1}=\tau_{1_{0}}$, Eqn. (8) has a pair of purely imaginary roots $\pm i \omega^{*}$ for $\tau_{2} \in\left[0, \tau_{2_{0}}\right)$.

In the following, we assume that

$$
(H 5)\left[\frac{\mathrm{d}(\operatorname{Re} \lambda)}{\mathrm{d} \tau_{1}}\right]_{\lambda=i \omega^{*}} \neq 0 .
$$

Thus, by the general Hopf bifurcation theorem for FDEs by Hale (1977), we have the following result on the stability and Hopf bifurcation in the system (5).

Theorem 1. For the system (5), assume that (H1), (H2), (H4) and (H5) are satisfied, and $\tau_{2} \in\left[0, \tau_{2_{0}}\right)$. Then the positive equilibrium $E_{0}\left(x^{*}, y^{*}\right)$ is asymptotically stable when $\tau_{1} \in\left(0, \tau_{1_{0}}\right)$, and the system (5) undergoes a Hopf bifurcation at the positive equilibrium $E_{0}\left(x^{*}, y^{*}\right)$ when $\tau_{1}=\tau_{1_{0}}$.

\section{Direction and stability of Hopf bifurcation}

In the previous section, we obtained conditions for Hopf bifurcation to occur when $\tau_{1}=\tau_{1_{0}}$. In this section, we shall derive explicit formulae determining the direction, stability, and period of these periodic solutions bifurcating from the positive equilibrium $E_{0}\left(x^{*}, y^{*}\right)$ at these critical values of $\tau_{1}$, by using techniques from normal form and center manifold theory (Hassard et al., 1981). Throughout this section, we always assume that the system (5) undergoes Hopf bifurcation at the positive equilibrium $E_{0}\left(x^{*}, y^{*}\right)$ for $\tau_{1}=\tau_{1_{0}}$, and then $\pm i \omega^{*}$ denotes the corresponding purely imaginary roots of the characteristic equation at the positive equilibrium $E_{0}\left(x^{*}, y^{*}\right)$.

Without loss of generality, we assume that $\tau_{2}^{*}<\tau_{1_{0}}$, where $\tau_{2}^{*} \in\left(0, \tau_{2_{0}}\right)$. For convenience, let $\bar{u}_{i}(t)=$ $u_{i}(\tau t)(i=1,2)$ and $\tau_{1}=\tau_{1_{0}}+\mu$, where $\tau_{1_{0}}$ is defined by (17) and $\mu \in \mathbb{R}$; dropping the bar for the simplification of notation, then the system (5) can be written as an FDE in $C=C\left([-1,0], \mathbb{R}^{2}\right)$ as

$$
\dot{u}(t)=L_{\mu}\left(u_{t}\right)+F\left(\mu, u_{t}\right),
$$

where $u(t)=(x(t), y(t))^{T} \in C$ and $u_{t}(\theta)=u(t+\theta)=$ $(x(t+\theta), y(t+\theta))^{T} \in C$, and $L_{\mu}: C \rightarrow \mathbb{R}, F: \mathbb{R} \times C \rightarrow$ $\mathbb{R}$ are given by

$$
\begin{gathered}
L_{\mu} \phi=\left(\tau_{1}+\mu\right) C\left(\begin{array}{c}
\phi_{1}\left(-\frac{\tau_{2}^{*}}{\tau_{1_{0}}}\right. \\
\tau_{2}^{*} \\
\phi_{2}\left(-\frac{\tau_{1_{0}}}{\tau_{1}}\right)
\end{array}\right) \\
+\left(\tau_{1}+\mu\right) D\left(\begin{array}{c}
\phi_{1}(-1) \\
\phi_{2}(-1)
\end{array}\right)
\end{gathered}
$$

and

$$
F(\mu, \phi)=\left(\tau_{1}+\mu\right)\left(f_{1}, f_{2}\right)^{T},
$$

respectively, where $\phi(\theta)=\left(\phi_{1}(\theta), \phi_{2}(\theta)\right)^{T} \in C$,

$$
C=\left(\begin{array}{cc}
0 & m_{3} \\
n_{2} & 0
\end{array}\right), \quad D=\left(\begin{array}{cc}
m_{2} & 0 \\
0 & n_{3}
\end{array}\right)
$$

and

$$
\begin{aligned}
& f_{1}=m_{4} \phi_{1}(0) \phi_{1}(-1)+m_{5} \phi_{1}(0) \phi_{2}\left(-\frac{\tau_{2}^{*}}{\tau_{1_{0}}}\right), \\
& f_{2}=n_{4} \phi_{1}\left(-\frac{\tau_{2}^{*}}{\tau_{1_{0}}}\right) \phi_{2}(0)+n_{5} \phi_{2}(0) \phi_{2}(-1) .
\end{aligned}
$$

From the discussion in Section 2, we know that if $\mu=0$, then system (18) undergoes Hopf bifurcation at the positive equilibrium $E_{0}\left(x^{*}, y^{*}\right)$ and the associated characteristic equation of the system (18) has a pair of simple imaginary roots $\pm i \omega^{*} \tau_{1_{0}}$.

By the representation theorem, there is a matrix function with bounded variation components $\eta(\theta, \mu), \theta \in$ $[-1,0]$ such that

$$
L_{\mu} \phi=\int_{-1}^{0} \mathrm{~d} \eta(\theta, \mu) \phi(\theta), \quad \text { for } \phi \in C .
$$


In fact, we can choose

$$
\eta(\theta, \mu)=\left\{\begin{array}{lc}
\left(\tau_{1_{0}}+\mu\right)(C+D), & \theta=0, \\
\left(\tau_{1_{0}}+\mu\right)(C+D), & \theta \in\left[-\frac{\tau_{2}^{*}}{\tau_{1_{0}}}, 0\right), \\
\left(\tau_{1_{0}}+\mu\right) D, & \theta \in\left(-1,-\frac{\tau_{2}^{*}}{\tau_{1_{0}}}\right), \\
0, & \theta=-1 .
\end{array}\right.
$$

For $\phi \in C\left([-1,0], \mathbb{R}^{2}\right)$, define

$$
A(\mu) \phi=\left\{\begin{array}{lc}
\frac{\mathrm{d} \phi(\theta)}{\mathrm{d} \theta}, & -1 \leq \theta<0, \\
\int_{-1}^{0} \mathrm{~d} \eta(s, \mu) \phi(s), & \theta=0
\end{array}\right.
$$

and

$$
R \phi=\left\{\begin{array}{lc}
0, & -1 \leq \theta<0, \\
F(\mu, \phi), & \theta=0 .
\end{array}\right.
$$

Then (18) is equivalent to the abstract differential equation

$$
\dot{u}_{t}=A(\mu) u_{t}+R(\mu) u_{t}
$$

where $u_{t}(\theta)=u(t+\theta), \theta \in[-1,0]$.

For $\psi \in C\left([-1,0],\left(\mathbb{R}^{2}\right)^{*}\right)$, define

$$
A^{*} \psi(s)= \begin{cases}-\frac{\mathrm{d} \psi(s)}{\mathrm{d} s}, & s \in(0,1], \\ \int_{-1}^{0} \mathrm{~d} \eta^{T}(t, 0) \psi(-t), & s=0 .\end{cases}
$$

For $\phi \in C\left([-1,0], \mathbb{R}^{2}\right)$ and $\psi \in C\left([0,1],\left(\mathbb{R}^{2}\right)^{*}\right)$, define the bilinear form

$$
\langle\psi, \phi\rangle=\bar{\psi}(0) \phi(0)-\int_{-1}^{0} \int_{\xi=0}^{\theta} \psi^{T}(\xi-\theta) \mathrm{d} \eta(\theta) \phi(\xi) \mathrm{d} \xi
$$

where $\eta(\theta)=\eta(\theta, 0)$, the $A=A(0)$ and $A^{*}$ are adjoint operators. From the discussions in Section 2, we know that $\pm i \omega^{*} \tau_{1_{0}}$ are eigenvalues of $A(0)$, and they are also the eigenvalues of $A^{*}$ corresponding to $i \omega^{*} \tau_{1_{0}}$ and $-i \omega^{*} \tau_{1_{0}}$, respectively. By direct computation, we can obtain

$$
q(\theta)=(1, \alpha)^{T} e^{i \omega^{*} \tau_{1_{0}} \theta}, \quad q^{*}(s)=M\left(1, \alpha^{*}\right) e^{i \omega^{*} \tau_{1_{0}} s},
$$

$M=1 / K$, where

$$
\begin{aligned}
\alpha= & \frac{i \omega^{*}-m_{2} e^{-i \omega^{*} \tau_{1_{0}}}}{m_{3} e^{-i \omega^{*} \tau_{2}^{*}}}, \\
\alpha^{*}= & -\frac{i \omega^{*}+m_{2} e^{-i \omega^{*} \tau_{1_{0}}}}{n_{2} e^{-i \omega^{*} \tau_{2}^{*}}}, \\
K= & 1+\bar{\alpha} \alpha^{*}+m_{2} \tau_{1_{0}} e^{i \omega^{*} \tau_{1_{0}}}+n_{2} \alpha^{*} \tau_{2}^{*} e^{i \omega^{*} \tau_{2}^{*}} \\
& +m_{3} \bar{\alpha} \tau_{2}^{*} e^{i \omega^{*} \tau_{2}^{*}}+n_{3} \bar{\alpha} \alpha^{*} \tau_{1_{0}} e^{i \omega^{*} \tau_{1_{0}}} .
\end{aligned}
$$

Furthermore, $\left\langle q^{*}(s), q(\theta)\right\rangle=1$ and $\left\langle q^{*}(s), \bar{q}(\theta)\right\rangle=0$.
Next, we use the same notation as Hassard et al. (1981), and we first compute the coordinates to describe the center manifold $C_{0}$ at $\mu=0$. Let $u_{t}$ be the solution of Eqn. (18) when $\mu=0$.

Define

$$
\begin{aligned}
z(t) & =\left\langle q^{*}, u_{t}\right\rangle, \\
W(t, \theta) & =u_{t}(\theta)-2 \operatorname{Re}\{z(t) q(\theta)\} .
\end{aligned}
$$

on the center manifold $C_{0}$. We have

$$
W(t, \theta)=W(z(t), \bar{z}(t), \theta),
$$

where

$$
\begin{aligned}
W(z(t), \bar{z}(t), \theta) & =W(z, \bar{z}) \\
& =W_{20} \frac{z^{2}}{2}+W_{11} z \bar{z}+W_{02} \frac{\bar{z}^{2}}{2}+\cdots,
\end{aligned}
$$

and $z$ and $\bar{z}$ are local coordinates for center manifold $C_{0}$ in the directions of $q^{*}$ and $\bar{q}^{*}$. Noting that $W$ is real if so is $u_{t}$, we consider only real solutions. For solutions $u_{t} \in C_{0}$ of (18),

$$
\begin{aligned}
& \dot{z}(t)=i \omega^{*} \tau_{1_{0}} z+\bar{q}^{*}(\theta) F(0, W(z, \bar{z}, \theta)+2 \operatorname{Re}\{z q(\theta)\} \\
& \stackrel{\text { def }}{=} i \omega^{*} \tau_{1_{0}} z+\bar{q}^{*}(0) F_{0} .
\end{aligned}
$$

In other words,

$$
\dot{z}(t)=i \omega^{*} \tau_{1_{0}} z+g(z, \bar{z})
$$

where

$$
g(z, \bar{z})=g_{20} \frac{z^{2}}{2}+g_{11} z \bar{z}+g_{02} \frac{\bar{z}^{2}}{2}+g_{21} \frac{z^{2} \bar{z}}{2}+\cdots .
$$

Hence, we have

$$
\begin{aligned}
g(z, \bar{z}) & =\bar{q}^{*}(0) F_{0}(z, \bar{z})=F\left(0, u_{t}\right) \\
= & \bar{M} \tau_{1_{0}}\left[\left(m_{4} e^{-i \omega^{*} \tau_{1_{0}}}+m_{5} \alpha e^{-i \omega^{*} \tau_{2}^{*}}\right)\right. \\
& \left.+\bar{\alpha}^{*}\left(n_{4} \alpha e^{-i \omega^{*} \tau_{2}^{*}}+n_{5} \alpha e^{-i \omega^{*} \tau_{1_{0}}}\right)\right] z^{2} \\
& +2 \bar{M} \tau_{1_{0}}\left[m_{4} \operatorname{Re}\left\{\alpha e^{-i \omega^{*} \tau_{1_{0}}}\right\}+m_{5} \operatorname{Re}\left\{\alpha e^{-i \omega^{*} \tau_{2}^{*}}\right\}\right. \\
& \left.+\bar{\alpha}^{*}\left(n_{4} \operatorname{Re}\left\{\alpha e^{i \omega^{*} \tau_{2}^{*}}\right\}+n_{5} \operatorname{Re}\left\{|\alpha|^{2} e^{i \omega^{*} \tau_{1_{0}}}\right\}\right)\right] z \bar{z} \\
& +\bar{M} \tau_{1_{0}}\left[m_{4} e^{i \omega^{*} \tau_{2}^{*}}+m_{5} \bar{\alpha} e^{i \omega^{*} \tau_{2}^{*}}+\bar{\alpha}^{*}\left(n_{4} \bar{\alpha} e^{i \omega^{*} \tau_{1_{0}}}\right.\right. \\
& \left.\left.+n_{5} \bar{\alpha}^{2} e^{i \omega^{*} \tau_{1_{0}}}\right)\right] \bar{z}^{2}+\bar{M} \tau_{1_{0}}\left\{m _ { 4 } \left(\frac{1}{2} W_{20}^{(1)}(0) \bar{\alpha} e^{i \omega^{*} \tau_{1_{0}}}\right.\right.
\end{aligned}
$$




$$
\begin{aligned}
& \left.+\frac{1}{2} W_{20}^{(2)}(-1)+\alpha W_{11}^{(1)}(0) e^{-i \omega^{*} \tau_{1_{0}}}+W_{11}^{(2)}(-1)\right) \\
& +m_{5}\left(\frac{1}{2} W_{20}^{(1)}(0) \bar{\alpha} e^{i \omega^{*} \tau_{2}^{*}}+W_{11}^{(1)}(0) \alpha e^{i \omega^{*} \tau_{2}^{*}}\right. \\
& \left.+W_{11}^{(2)}\left(-\frac{\tau_{2}^{*}}{\tau_{1_{0}}}\right)+\frac{1}{2} W_{20}^{(2)}\left(-\frac{\tau_{2}^{*}}{\tau_{1_{0}}}\right)\right) \\
& +\bar{\alpha}^{*}\left[n _ { 4 } \left(\frac{1}{2} W_{20}^{(1)}\left(-\frac{\tau_{2}^{*}}{\tau_{1_{0}}}\right) \bar{\alpha}+W_{11}^{(1)}\left(-\frac{\tau_{2}^{*}}{\tau_{1_{0}}}\right) \alpha\right.\right. \\
& \left.+W_{20}^{(2)}(0) e^{-i \omega^{*} \tau_{1_{0}}}+W_{11}^{(2)}(0) e^{-i \omega^{*} \tau_{2}^{*}}\right) \\
& +n_{5}\left(\frac{1}{2} W_{20}^{(2)}(0) \bar{\alpha} e^{i \omega^{*} \tau_{1_{0}}}+W_{11}^{(2)}(-1) \alpha\right. \\
& \left.\left.\left.+\alpha W_{11}^{(2)}(0) e^{i \omega^{*} \tau_{1_{0}}}+\frac{1}{2} W_{20}^{(2)}(-1) \bar{\alpha}\right)\right]\right\} z^{2} \bar{z}+\cdots
\end{aligned}
$$

Then we obtain

$$
\begin{aligned}
& g_{20}=2 \bar{M} \tau_{1_{0}}\left[\left(m_{4} e^{-i \omega^{*} \tau_{1_{0}}}+m_{5} \alpha e^{-i \omega^{*} \tau_{2}^{*}}\right)\right. \\
& \left.+\bar{\alpha}^{*}\left(n_{4} \alpha e^{-i \omega^{*} \tau_{2}^{*}}+n_{5} \alpha e^{-i \omega^{*} \tau_{1_{0}}}\right)\right], \\
& g_{11}=2 \bar{M} \tau_{1_{0}}\left[m_{4} \operatorname{Re}\left\{\alpha e^{-i \omega^{*} \tau_{1_{0}}}\right\}+m_{5} \operatorname{Re}\left\{\alpha e^{-i \omega^{*} \tau_{2}^{*}}\right\}\right. \\
& \left.+\bar{\alpha}^{*}\left(n_{4} \operatorname{Re}\left\{\alpha e^{i \omega^{*} \tau_{2}^{*}}\right\}+n_{5} \operatorname{Re}\left\{|\alpha|^{2} e^{i \omega^{*} \tau_{1_{0}}}\right\}\right)\right], \\
& g_{02}=2 \bar{M} \tau_{1_{0}}\left[m_{4} e^{i \omega^{*} \tau_{2}^{*}}+m_{5} \bar{\alpha} e^{i \omega^{*} \tau_{2}^{*}}\right. \\
& \left.+\bar{\alpha}^{*}\left(n_{4} \bar{\alpha} e^{i \omega^{*} \tau_{1_{0}}}+n_{5} \bar{\alpha}^{2} e^{i \omega^{*} \tau_{1_{0}}}\right)\right], \\
& g_{21}=2 \bar{M} \tau_{1_{0}}\left\{m _ { 4 } \left(\frac{1}{2} W_{20}^{(1)}(0) \bar{\alpha} e^{i \omega^{*} \tau_{1_{0}}}+\frac{1}{2} W_{20}^{(2)}(-1)\right.\right. \\
& \left.+\alpha W_{11}^{(1)}(0) e^{-i \omega^{*} \tau_{1_{0}}}+W_{11}^{(2)}(-1)\right) \\
& +m_{5}\left(\frac{1}{2} W_{20}^{(1)}(0) \bar{\alpha} e^{i \omega^{*} \tau_{2}^{*}}+W_{11}^{(1)}(0) \alpha e^{-i \omega^{*} \tau_{2}^{*}}\right. \\
& \left.+W_{11}^{(2)}\left(-\frac{\tau_{2}^{*}}{\tau_{1_{0}}}\right)+\frac{1}{2} W_{20}^{(2)}\left(-\frac{\tau_{2}^{*}}{\tau_{1_{0}}}\right)\right) \\
& +\bar{\alpha}^{*}\left[n _ { 4 } \left(\frac{1}{2} W_{20}^{(1)}\left(-\frac{\tau_{2}^{*}}{\tau_{1_{0}}}\right) \bar{\alpha}+W_{11}^{(1)}\left(-\frac{\tau_{2}^{*}}{\tau_{1_{0}}}\right) \alpha\right.\right. \\
& \left.+\frac{1}{2} W_{20}^{(2)}(0) e^{i \omega^{*} \tau_{2}^{*}}+W_{11}^{(2)}(0) e^{-i \omega^{*} \tau_{2}^{*}}\right) \\
& +n_{5}\left(\frac{1}{2} W_{20}^{(2)}(0) \bar{\alpha} e^{i \omega^{*} \tau_{1}}+W_{11}^{(2)}(-1) \alpha\right. \\
& \left.\left.\left.+\alpha W_{11}^{(2)}(0) e^{-i \omega^{*} \tau_{1_{0}}}+\frac{1}{2} W_{20}^{(2)}(-1) \bar{\alpha}\right)\right]\right\} .
\end{aligned}
$$

For unknown $W_{20}^{(i)}(\theta), W_{11}^{(i)}(\theta), i=1,2$ in $g_{21}$, we still have to compute them. From (25) and (26), we have

$$
\begin{aligned}
W^{\prime} & = \begin{cases}A W-2 \operatorname{Re}\left\{\bar{q}^{*}(0) F_{0} q(\theta)\right\}, & -1 \leq \theta<0, \\
A W-2 \operatorname{Re}\left\{\bar{q}^{*}(0) F_{0} q(\theta)\right\}+F_{0}, & \theta=0\end{cases} \\
& =A W+H(z, \bar{z}, \theta),
\end{aligned}
$$

where

$$
\begin{aligned}
H(z, \bar{z}, \theta)= & H_{20}(\theta) \frac{z^{2}}{2}+H_{11}(\theta) z \bar{z} \\
& +H_{02}(\theta) \frac{\bar{z}^{2}}{2}+\ldots
\end{aligned}
$$

Comparing the coefficients, we obtain

$$
\begin{aligned}
\left(A W-2 i \tau_{1_{0}} \omega^{*}\right) W_{20} & =-H_{20}(\theta), \\
A W_{11}(\theta) & =-H_{11}(\theta) .
\end{aligned}
$$

We know that for $\theta \in[-1,0)$,

$$
\begin{aligned}
H(z, \bar{z}, \theta) & =-\bar{q}^{*}(0) f_{0} q(\theta)-q^{*}(0) \overline{f_{0}} \bar{q}(\theta) \\
& =-g(z, \bar{z}) q(\theta)-\bar{g}(z, \bar{z}) \bar{q}(\theta) .
\end{aligned}
$$

Comparing the coefficients of (33) with (30) gives

$$
\begin{aligned}
& H_{20}(\theta)=-g_{20} q(\theta)-\bar{g}_{02} \bar{q}(\theta), \\
& H_{11}(\theta)=-g_{11} q(\theta)-\bar{g}_{11} \bar{q}(\theta) .
\end{aligned}
$$

From (31), (34) and the definition of $A$, we get

$$
\dot{W}_{20}(\theta)=2 i \omega^{*} \tau_{1_{0}} W_{20}(\theta)+g_{20} q(\theta)+g_{02} \bar{q}(\theta) .
$$

Noting that $q(\theta)=q(0) e^{i \omega^{*} \tau_{1_{0}} \theta}$, we have

$$
\begin{aligned}
W_{20}(\theta)= & \frac{i g_{20}}{\omega^{*} \tau_{1_{0}}} q(0) e^{i \omega^{*} \tau_{1_{0}} \theta}+\frac{i \bar{g}_{02}}{3 \omega^{*} \tau_{1_{0}}} \bar{q}(0) e^{-i \omega^{*} \tau_{1_{0}} \theta} \\
& +E_{1} e^{2 i \omega^{*} \tau_{1_{0}} \theta}
\end{aligned}
$$

where $E_{1}=\left(E_{1}^{(1)}, E_{1}^{(2)}\right) \in \mathbb{R}^{2}$ is a constant vector. Similarly, from (32), (35) and the definition of $A$, we have

$$
\begin{aligned}
\dot{W}_{11}(\theta)= & g_{11} q(\theta)+g_{11}^{-} \bar{q}(\theta), \\
W_{11}(\theta)= & -\frac{i g_{11}}{\omega^{*} \tau_{1_{0}}} q(0) e^{i \omega^{*} \tau_{1_{0}} \theta} \\
& +\frac{i \bar{g}_{11}}{\omega^{*} \tau_{1_{0}}} \bar{q}(0) e^{-i \omega^{*} \tau_{1_{0}} \theta}+E_{2},
\end{aligned}
$$

where $E_{2}=\left(E_{2}^{(1)}, E_{2}^{(2)}\right) \in \mathbb{R}^{2}$ is a constant vector.

In what follows, we shall seek appropriate $E_{1}, E_{2}$ in (37), (39), respectively. From the definition of $A$ and (34), (35) it follows that

$$
\int_{-1}^{0} \mathrm{~d} \eta(\theta) W_{20}(\theta)=2 i \omega^{*} \tau_{1_{0}} W_{20}(0)-H_{20}(0)
$$

and

$$
\int_{-1}^{0} \mathrm{~d} \eta(\theta) W_{11}(\theta)=-H_{11}(0),
$$

where $\eta(\theta)=\eta(0, \theta)$. From (31), we have

$$
\begin{aligned}
& H_{20}(0)=-g_{20} q(0)-g_{02} \bar{q}(0)+2 \tau_{1_{0}}\left(H_{1}, H_{2}\right)^{T}, \\
& H_{11}(0)=-g_{11} q(0)-g_{11}(0) \bar{q}(0)+2 \tau_{1_{0}}\left(P_{1}, P_{2}\right)^{T},
\end{aligned}
$$


where

$$
\begin{aligned}
& H_{1}=m_{4} e^{-i \omega^{*} \tau_{1_{0}}}+m_{5} \alpha e^{-i \omega^{*} \tau_{2}^{*}}, \\
& H_{2}=n_{4} \alpha e^{-i \omega^{*} \tau_{2}^{*}}+n_{5} \alpha e^{-i \omega^{*} \tau_{1_{0}}}, \\
& P_{1}=m_{4} \operatorname{Re}\left\{\alpha e^{-i \omega^{*} \tau_{1_{0}}}\right\}+m_{5} \operatorname{Re}\left\{\alpha e^{-i \omega^{*} \tau_{2}^{*}}\right\}, \\
& P_{2}=n_{4} \operatorname{Re}\left\{\alpha e^{i \omega^{*} \tau_{2}^{*}}\right\}+n_{5} \operatorname{Re}\left\{|\alpha|^{2} e^{i \omega^{*} \tau_{1_{0}}}\right\} .
\end{aligned}
$$

Noting that

$$
\begin{gathered}
\left(i \omega^{*} \tau_{1_{0}} I-\int_{-1}^{0} e^{i \omega^{*} \tau_{1_{0}} \theta} \mathrm{d} \eta(\theta)\right) q(0)=0 \\
\left(-i \omega^{*} \tau_{1_{0}} I-\int_{-1}^{0} e^{-i \omega^{*} \tau_{1_{0}} \theta} \mathrm{d} \eta(\theta)\right) \bar{q}(0)=0
\end{gathered}
$$

and substituting (37) and (42) into (40), we have

$$
\left(2 i \omega^{*} \tau_{1_{0}} I-\int_{-1}^{0} e^{2 i \omega^{*} \tau_{1_{0}} \theta} \mathrm{d} \eta(\theta)\right) E_{1}=2 \tau_{1_{0}}\left(H_{1}, H_{2}\right)^{T} .
$$

That is,

$$
\begin{aligned}
& \left(\begin{array}{cc}
2 i \omega^{*}-m_{2} e^{-2 i \omega^{*} \tau_{1_{0}}} & -m_{3} e^{-2 i \omega^{*} \tau_{2}^{*}} \\
-n_{2} e^{-2 i \omega^{*} \tau_{2}^{*}} & 2 i \omega^{*}-n_{3} e^{-2 i \omega^{*} \tau_{1_{0}}}
\end{array}\right) E_{1} \\
& =2\left(H_{1}, H_{2}\right)^{T} \text {. }
\end{aligned}
$$

It follows that

$$
E_{1}^{(1)}=\frac{\Delta_{11}}{\Delta_{1}}, \quad E_{1}^{(2)}=\frac{\Delta_{12}}{\Delta_{1}},
$$

where

$$
\begin{gathered}
\Delta_{1}=\operatorname{det}\left(\begin{array}{ll}
v_{1} & v_{2} \\
v_{3} & v_{4}
\end{array}\right), \\
\Delta_{11}=2 \operatorname{det}\left(\begin{array}{ll}
H_{1} & v_{2} \\
H_{2} & v_{4}
\end{array}\right), \\
\Delta_{12}=2 \operatorname{det}\left(\begin{array}{ll}
v_{1} & H_{1} \\
v_{2} & H_{2}
\end{array}\right),
\end{gathered}
$$

where

$$
\begin{aligned}
& v_{1}=2 i \omega^{*}-m_{2} e^{-2 i \omega^{*} \tau_{1_{0}}}, \quad v_{2}=-m_{3} e^{-2 i \omega^{*} \tau_{2}^{*}}, \\
& v_{3}=-n_{2} e^{-2 i \omega^{*} \tau_{2}^{*}} \\
& v_{4}=2 i \omega^{*}-n_{3} e^{-2 i \omega^{*} \tau_{1_{0}}} .
\end{aligned}
$$

Similarly, substituting (38) and (43) into (41), we have

$$
\left(\int_{-1}^{0} \mathrm{~d} \eta(\theta)\right) E_{2}=2 \tau_{1_{0}}\left(P_{1}, P_{2}\right)^{T} \text {. }
$$

That is,

$$
\left(\begin{array}{cc}
m_{2} & m_{3} \\
n_{2} & n_{3}
\end{array}\right) E_{2}=2\left(-P_{1},-P_{2}\right)^{T}
$$

It follows that

$$
E_{2}^{(1)}=\frac{\Delta_{21}}{\Delta_{2}}, \quad E_{2}^{(2)}=\frac{\Delta_{22}}{\Delta_{2}},
$$

where

$$
\begin{aligned}
\Delta_{2} & =\operatorname{det}\left(\begin{array}{cc}
m_{2} & m_{3} \\
n_{2} & n_{3}
\end{array}\right), \\
\Delta_{21} & =2 \operatorname{det}\left(\begin{array}{cc}
-P_{1} & m_{3} \\
-P_{2} & n_{3}
\end{array}\right), \\
\Delta_{22} & =2 \operatorname{det}\left(\begin{array}{cc}
m_{2} & -P_{1} \\
n_{2} & -P_{2}
\end{array}\right) .
\end{aligned}
$$

From (37), (39), (44), (45), we can calculate $g_{21}$ and derive the following values:

$$
\begin{aligned}
c_{1}(0) & =\frac{i}{2 \omega^{*} \tau_{1_{0}}}\left(g_{20} g_{11}-2\left|g_{11}\right|^{2}-\frac{\left|g_{02}\right|^{2}}{3}\right)+\frac{g_{21}}{2}, \\
\mu_{2} & =-\frac{\operatorname{Re}\left\{c_{1}(0)\right\}}{\operatorname{Re}\left\{\lambda^{\prime}\left(\tau_{1_{0}}\right)\right\}}, \\
\beta_{2} & =2 \operatorname{Re}\left(c_{1}(0)\right), \\
T_{2} & =-\frac{\operatorname{Im}\left\{c_{1}(0)\right\}+\mu_{2} \operatorname{Im}\left\{\lambda^{\prime}\left(\tau_{1_{0}}\right)\right\}}{\omega^{*} \tau_{1_{0}}} .
\end{aligned}
$$

These formulas give a description of the Hopf bifurcation periodic solutions of (18) at $\tau=\tau_{1_{0}}$ on the center manifold. From the discussion above, we have the following result.

Theorem 2. For the system (5), assume that (H1), (H2), (H4) and (H5) are satisfied. The periodic solution is supercritical (resp. subcritical) if $\mu_{2}>0$ (resp. $\left.\mu_{2}<0\right)$. The bifurcating periodic solutions are orbitally asymptotically stable with an asymptotical phase (resp. unstable) if $\beta_{2}<0$ (resp. $\beta_{2}>0$ ). The period of the bifurcating periodic solutions increases (resp. decrease) if $T_{2}>0$ (resp. $\left.T_{2}<0\right)$.

\section{Numerical examples}

In this section, we present some numerical results of the system (5) to verify the analytical predictions obtained in the previous section. From Section 3, we may determine the direction of a Hopf bifurcation and the stability of the bifurcation periodic solutions. Let us consider the following system:

$$
\left\{\begin{array}{l}
\dot{x}(t)=x(t)\left[0.5-0.5 x\left(t-\tau_{1}\right)-y\left(t-\tau_{2}\right)\right], \\
\dot{y}(t)=y(t)\left[-0.5+x\left(t-\tau_{2}\right)-y\left(t-\tau_{1}\right)\right]
\end{array}\right.
$$

which has a positive equilibrium $E_{0}\left(x^{*}, y^{*}\right)=\left(\frac{2}{3}, \frac{1}{6}\right)$. When $\tau_{1}=0$, we can easily obtain that $(H 2)$ and $(H 4)$ are satisfied. For example take $n=0$. By some computation by means of Matlab 7.0, we get $\omega_{0} \approx 0.4509, \tau_{2_{0}} \approx$ 
3.357. From Lemma 2, we know that the transversal condition is satisfied. Thus the positive equilibrium $E_{0}=$ $\left(\frac{2}{3}, \frac{1}{6}\right)$ is asymptotically stable for $\tau_{2}<\tau_{2_{0}} \approx 3.357$ and unstable for $\tau_{2}>\tau_{2_{0}} \approx 3.357$, which is shown in Fig. 1. When $\tau_{2}=\tau_{2_{0}} \approx 3.357$, Eqn. (46) undergoes Hopf bifurcation at the positive equilibrium $E_{0}=\left(\frac{2}{3}, \frac{1}{6}\right)$, i.e., a small amplitude periodic solution occurs around $E_{0}=\left(\frac{2}{3}, \frac{1}{6}\right)$ when $\tau_{1}=0$ and $\tau_{2}$ is close to $\tau_{2_{0}}=1.52$, which is shown in Fig. 2.

Let $\tau_{2}=3 \in(0,3.357)$ and choose $\tau_{1}$ as a parameter. We have $\tau_{1_{0}} \approx 0.6673$. Then the positive equilibrium is asymptotically stable when $\tau_{1} \in\left[0, \tau_{1_{0}}\right)$. The Hopf bifurcation value of Eqn. (46) is $\tau_{1_{0}} \approx 0.6673$. By the algorithms derived in Section 3, we can obtain

$$
\begin{aligned}
\lambda^{\prime}\left(\tau_{1_{0}}\right) & \approx 0.3732-8.1075 i \\
c_{1}(0) & \approx-3.1532-4.4312 i \\
\mu_{2} & \approx 0.2335 \\
\beta_{2} & \approx-5.2312 \\
T_{2} & \approx 7.1239
\end{aligned}
$$

Furthermore, it follows that $\mu_{2}>0$ and $\beta_{2}<0$. Thus, the positive equilibrium $E_{0}=\left(\frac{2}{3}, \frac{1}{6}\right)$ is stable when $\tau_{1}<\tau_{1_{0}}$ as is illustrated by computer simulations (see Fig. 3). When $\tau_{1}$ passes through the critical value $\tau_{1_{0}}$, the positive equilibrium $E_{0}=\left(\frac{2}{3}, \frac{1}{6}\right)$ looses its stability and a Hopf bifurcation occurs, i.e., a family of periodic solutions bifurcate from the positive equilibrium $E_{0}=\left(\frac{2}{3}, \frac{1}{6}\right)$. Since $\mu_{2}>0$ and $\beta_{2}<0$, the direction of Hopf bifurcation is $\tau_{1}>\tau_{1_{0}}$, and these bifurcating periodic solutions from $E_{0}=\left(\frac{2}{3}, \frac{1}{6}\right)$ at $\tau_{1_{0}}$ are stable, which is depicted in Fig. 4.

\section{Conclusions}

In this paper, we investigated the local stability of the positive equilibrium $E_{0}\left(x^{*}, y^{*}\right)$ and local Hopf bifurcation of a Lotka-Volterra predator-prey model with two delays. We showed that, if $(H 1),(H 2),(H 4)$ and $(H 5)$ are satisfied, and $\tau_{2} \in\left[0, \tau_{2_{0}}\right)$, then the positive equilibrium $E_{0}\left(x^{*}, y^{*}\right)$ is asymptotically stable when $\tau_{1} \in$ $\left(0, \tau_{1_{0}}\right)$. As the delay $\tau_{1}$ increases, the positive equilibrium $E_{0}\left(x^{*}, y^{*}\right)$ loses its stability and a sequence of Hopf bifurcations occur at the positive equilibrium $E_{0}\left(x^{*}, y^{*}\right)$, i.e., a family of periodic orbits bifurcates from the positive equilibrium $E_{0}\left(x^{*}, y^{*}\right)$. Finally, the direction of Hopf bifurcation and the stability of the bifurcating periodic orbits were discussed by applying normal form theory and the center manifold theorem. A numerical example verifying our theoretical results was also given.

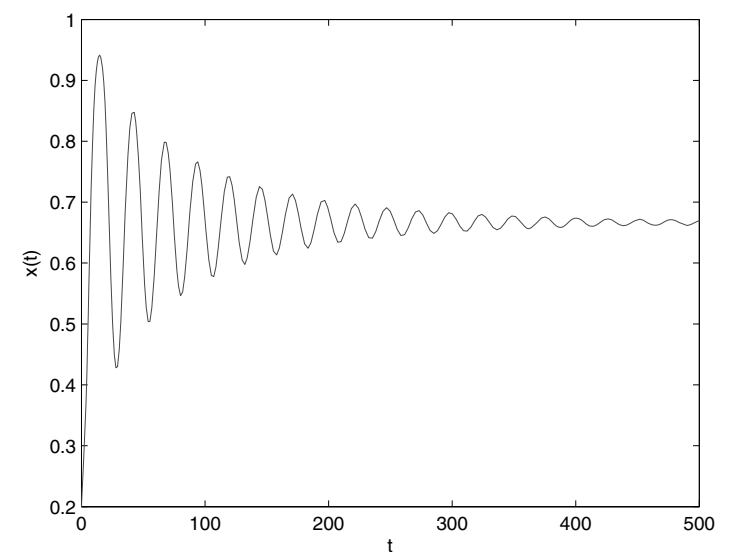

(a)

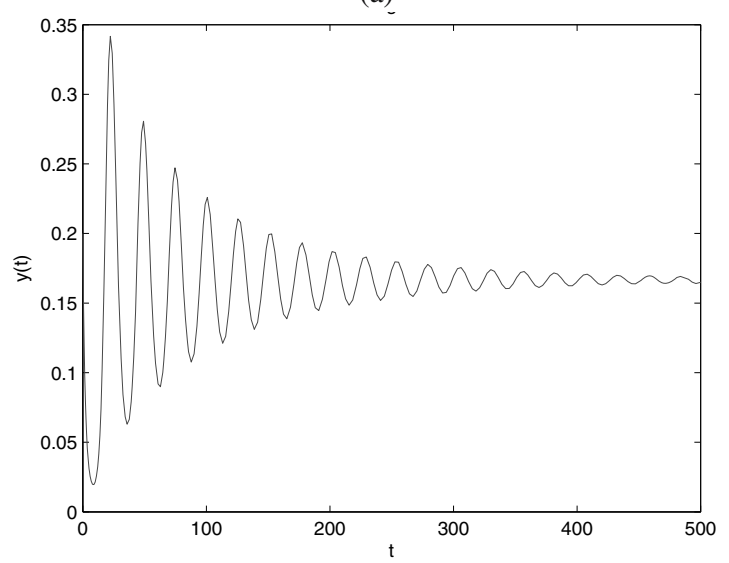

(b)

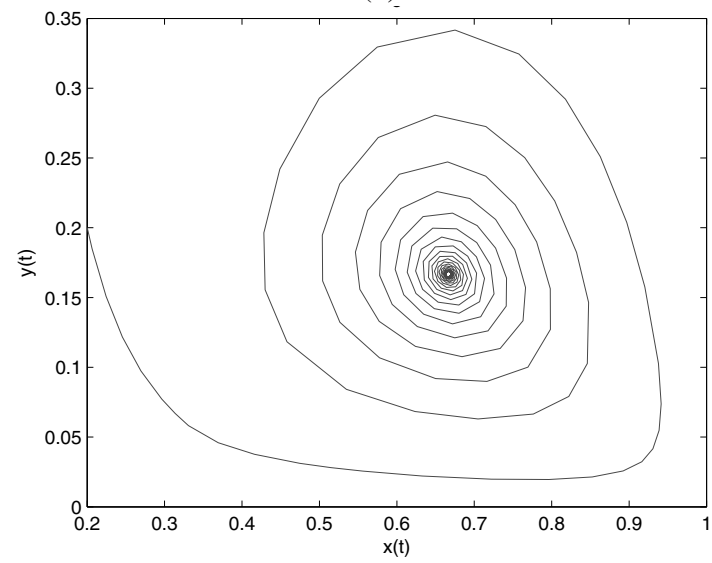

(c)

Fig. 1. Behavior and phase portrait of the system (46) with $\tau_{1}=$ $0, \tau_{2}=3.7>\tau_{2_{0}} \approx 3.357$. Hopf bifurcation occurs from the positive equilibrium $E_{0}=\left(\frac{2}{3}, \frac{1}{6}\right)$. The initial value is $(0.2,0.2)$.

\section{Acknowledgment}

We wish to thank the reviewers for their valuable comments that led to truly significant improvements in the manuscript. 


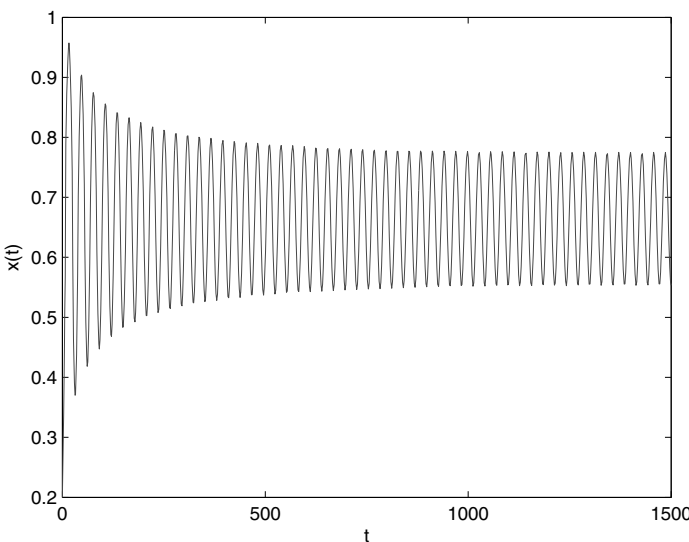

(a)

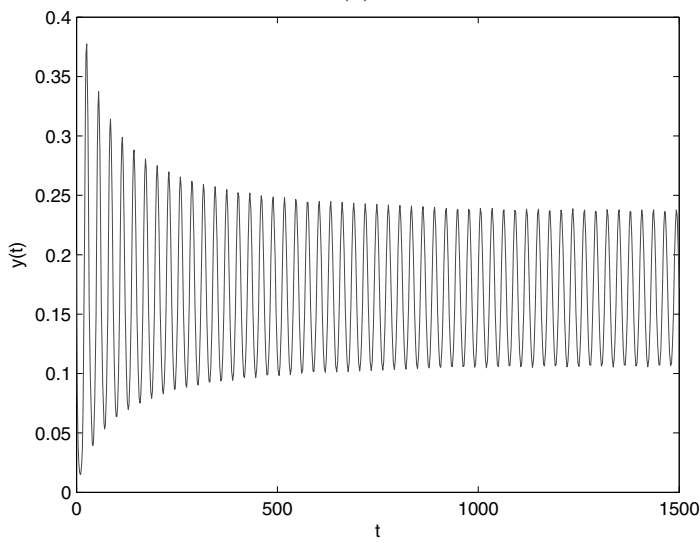

(b)

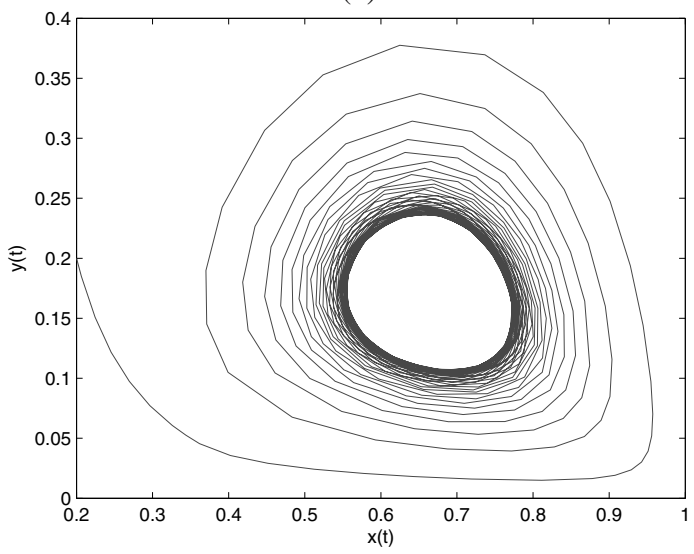

(c)

Fig. 2. Behavior and phase portrait of the system (46) with $\tau_{1}=$ $0, \tau_{2}=3<\tau_{2_{0}} \approx 3.357$. The positive equilibrium $E_{0}=\left(\frac{2}{3}, \frac{1}{6}\right)$ is asymptotically stable. The initial value is $(0.2,0.2)$

\section{References}

Bhattacharyya, R. and Mukhopadhyay, B. (2006). Spatial dynamics of nonlinear prey-predator models with prey migration and predator switching, Ecological Complexity 3(2): 160-169.

Faria, T. (2001). Stability and bifurcation for a delayed predator-

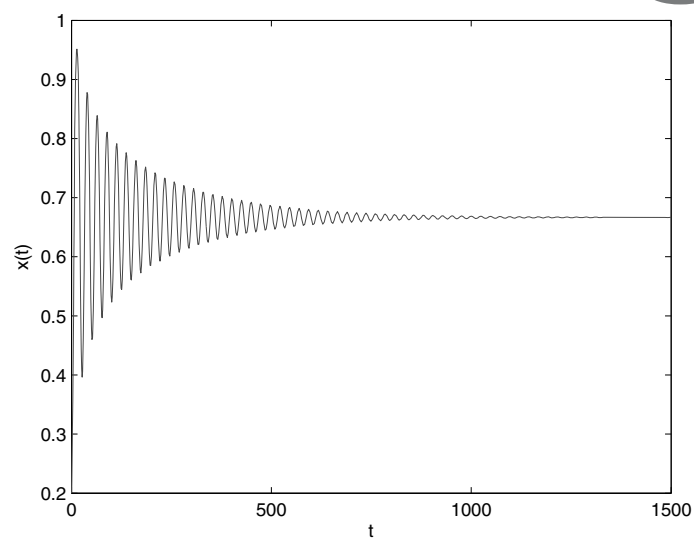

(a)

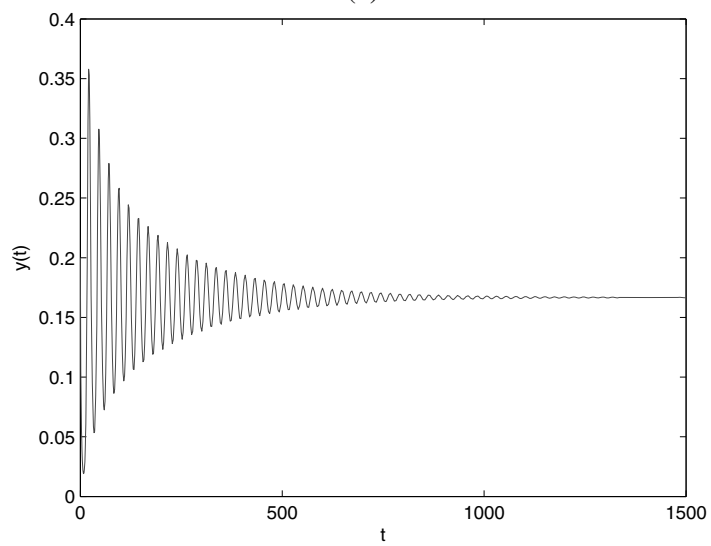

(b)

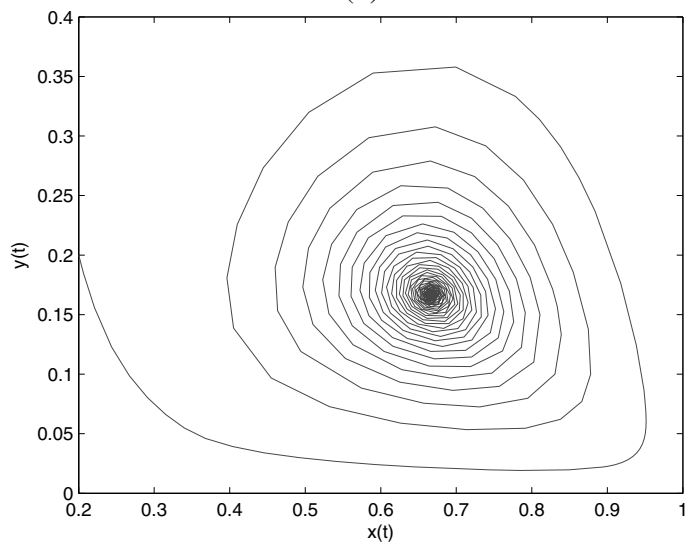

(c)

Fig. 3. Behavior and phase portrait of the system (46) with $\tau_{2}=$ $3, \tau_{1}=0.5<\tau_{2_{0}} \approx 0.6673$. The positive equilibrium $E_{0}=\left(\frac{2}{3}, \frac{1}{6}\right)$ is asymptotically stable. The initial value is $(0.2,0.2)$.

prey model and the effect of diffusion, Journal of Mathematical Analysis and Applications 254(2): 433-463.

Gao, S.J., Chen, L.S. and Teng, Z.D. (2008). Hopf bifurcation and global stability for a delayed predator-prey system with stage structure for predator, Applied Mathematics and Computation 202(2): 721-729. 


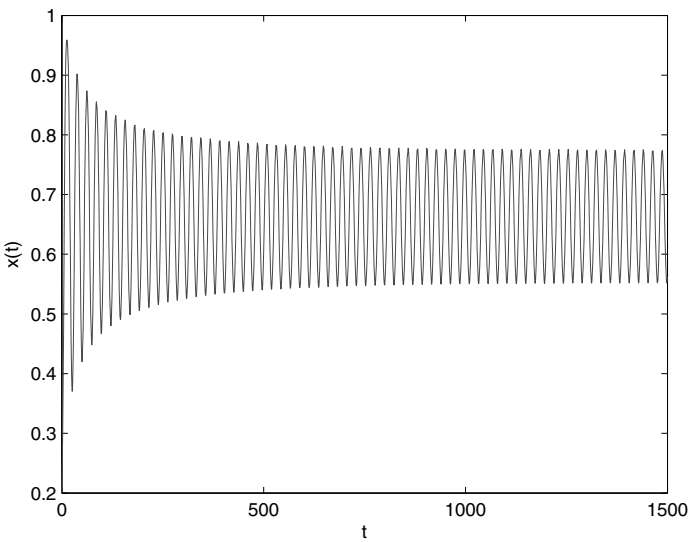

(a)

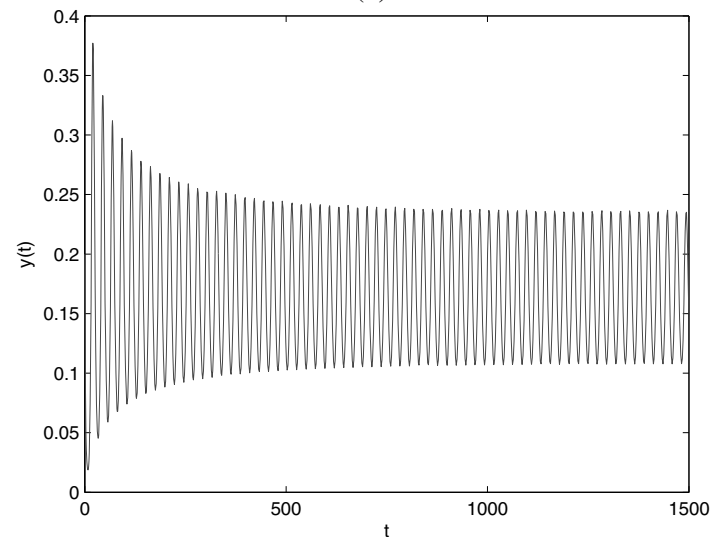

(b)

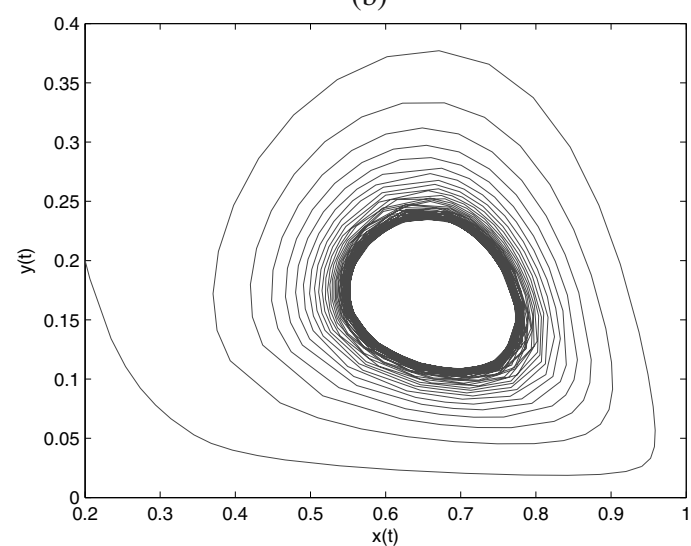

(c)

Fig. 4. Behavior and phase portrait of the system (46) with $\tau_{2}=$ $3, \tau_{1}=0.8>\tau_{1_{0}} \approx 0.6673$. Hopf bifurcation occurs from the positive equilibrium $E_{0}=\left(\frac{2}{3}, \frac{1}{6}\right)$. The initial value is $(0.2,0.2)$.

Hale, J. (1977). Theory of Functional Differential Equations, Springer-Verlag, Berlin.
Hassard, B., Kazarino, D. and Wan, Y. (1981). Theory and Applications of Hopf Bifurcation, Cambridge University Press, Cambridge.

Kar, T. and Pahari, U. (2007). Modelling and analysis of a preypredator system stage-structure and harvesting, Nonlinear Analysis: Real World Applications 8(2): 601-609.

Klamka, J. (1991). Controllability of Dynamical Systems, Kluwer, Dordrecht.

Kuang, Y. (1993). Delay Differential Equations with Applications in Population Dynamics, Academic Press, Boston, MA.

Kuang, Y. and Takeuchi, Y. (1994). Predator-prey dynamics in models of prey dispersal in two-patch environments, Mathematical Biosciences 120(1): 77-98.

Li, K. and Wei, J. (2009). Stability and Hopf bifurcation analysis of a prey-predator system with two delays, Chaos, Solitons \& Fractals 42(5): 2603-2613.

May, R.M. (1973). Time delay versus stability in population models with two and three trophic levels, Ecology 4(2): 315-325.

Prajneshu Holgate, P. (1987). A prey-predator model with switching effect, Journal of Theoretical Biology 125(1): 61-66.

Ruan, S. and Wei, J. (2003). On the zero of some transcendential functions with applications to stability of delay differential equations with two delays, Dynamics of Continuous, Discrete and Impulsive Systems Series A 10(1): 863-874.

Song, Y.L. and Wei, J. (2005). Local Hopf bifurcation and global periodic solutions in a delayed predator-prey system, Journal of Mathematical Analysis and Applications 301(1): $1-21$.

Teramoto, E.I., Kawasaki, K. and Shigesada, N. (1979). Switching effects of predaption on competitive prey species, Journal of Theoretical Biology 79(3): 303-315.

Xu, R., Chaplain, M.A.J. and Davidson F.A. (2004). Periodic solutions for a delayed predator-prey model of prey dispersal in two-patch environments, Nonlinear Analysis: Real World Applications 5(1): 183-206.

Xu, R. and Ma, Z.E. (2008). Stability and Hopf bifurcation in a ratio-dependent predator-prey system with stage structure, Chaos, Solitons \& Fractals 38(3): 669-684.

Yan, X.P. and Li, W.T. (2006). Hopf bifurcation and global periodic solutions in a delayed predator-prey system, Applied Mathematics and Computation 177(1): 427-445.

Yan, X.P. and Zhang, C.H. (2008). Hopf bifurcation in a delayed Lokta-Volterra predator-prey system, Nonlinear Analysis: Real World Applications 9(1): 114-127.

Zhou, X.Y., Shi, X.Y. and Song, X.Y. (2008). Analysis of nonautonomous predator-prey model with nonlinear diffusion and time delay, Applied Mathematics and Computation 196(1): 129-136. 


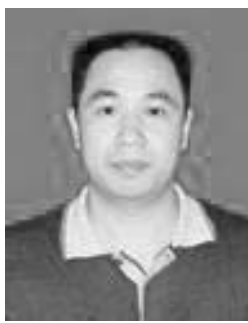

Changjin Xu received the M.Sc. degree from the Kunming University of Science and Technology, Kunming, China, in 2004, and the Ph.D. degree from Central South University, Changsha, China, in 2010. He is also a lecturer at the Faculty of Science, Hunan Institute of Engineering, Xiangtan, China. His research interests focus on the stability and bifurcation theory of differential equations with delays.

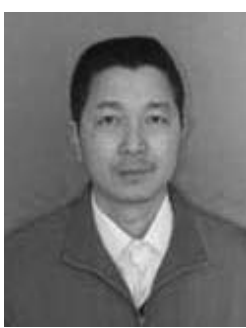

Maoxin Liao received the M.Sc. degree from the Changsha University of Science and Technology, Changsha, in 2005, and the Ph.D. degree from Central South University, Changsha, China, in 2010. He is also an associate professor at the School of Mathematics and Physics, University of South China, Hengyang, China. His research interests focus on the stability and bifurcation theory of differential equations with delays.

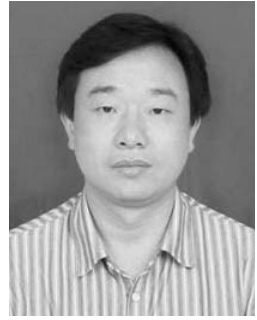

Xiaofei He is a Ph.D. student at the School of Mathematical Sciences and Computing Technology, Central South University, Changsha, China, and an associate professor at the College of Mathematical and Computer Science, Jishou University, Hunan Jishou, China. He received his M.S. from Hunan Normal University, Changsha, in 2008. His research interests focus on the stability and bifurcation theory of differential equations with delays.

Received: 16 July 2010

Revised: 8 October 2010 